Methodology Blood pressure (pressure-volume analysis or tail cuff plethysmography) was measured in wild type (WT) and Brn-3b knockout (KO) mice. Immunostaining and histological analysis were performed in the aorta of these animals. Gene expression analysis was performed using RNAseq and the expression pattern of Brn-3b was evaluated in primary VSMC cultures by immunofluorescence and qRT-PCR.

Results Brn-3b KO mice (2-6 months) developed spontaneous hypertension and vascular dysfunction such as neointimal hyperplasia, increased extracellular matrix (ECM) deposition and calcification. RNAseq analysis revealed that loss of Brn-3b in the aorta increased ECM gene expression, including collagens. Immunostaining of aortic sections from WT mice showed that Brn-3b was principally expressed in the tunica media, mostly composed of VSMC. Additionally, Brn-3b protein and mRNA were detectable in human and rodent primary VSMC cultures.

Conclusions Brn-3b loss in vivo is associated with vascular dysfunction and hypertension. Future studies will aim to investigate the role of Brn-3b in VSMC phenotypical changes.

\section{P30 EFFECTS OF CARNITINE SUPPLEMENTATION IN THE TYPE 1 DIABETIC HEART: AN IN VIVO HYPERPOLARIZED MRS STUDY}

Dragana Savic, Kerstin Timm, Vicky Ball, Lisa Heather, Damian Tyler. Department of Physiology, Anatomy and Genetics, University of Oxford

\subsection{6/heartjnl-2018-BSCR.35}

Carnitine transports long-chain fatty acids across the mitochondrial membrane for subsequent $\beta$-oxidation. It is known that carnitine levels are decreased in cardiac diseases, such as diabetes, and that carnitine supplementation can have cardioprotective effects.

The purpose of this study was to investigate the effects of l-carnitine supplementation on cardiac metabolism in the diabetic rat heart.

Type 1 diabetic rats were generated by streptozotocin injection, control rats were injected with citrate buffer and all were treated for 3 weeks with daily injections of either l-carnitine or saline, where after they were subjected to CINEMRI and hyperpolarized MRS.

Blood glucose levels were elevated in both diabetic groups, with the saline treated diabetic group showing a progressive increase in hyperglycaemia. Hyperpolarized MRS demonstrated reduction of pyruvate dehydrogenase (PDH) flux in the diabetic groups, but PDH flux was significantly higher in the 1carnitine treated diabetic group. Both lactate and alanine were significantly elevated in the animals treated with l-carnitine.

l-carnitine supplementation stabilises hyperglycaemia and increases the metabolism of pyruvate in the diabetic heart. 1carnitine provides a means to improve pyruvate metabolism in the diabetic heart.

\section{P31 EFFECT OF GLYCOGEN CONTENT ON KETONE BODY OXIDATION AND GLYCOLYSIS IN THE ISOLATED RAT HEART}

Azrul Abdul Kadir, Cher-Rin Chong, Henry Lee, Brianna Stubbs, Pete Cox, Kieran Clarke, Rhys Evans. Department of Physiology, Anatomy and Genetics - University of Oxford

10.1136/heartjnl-2018-BSCR.36
Apart from acting as an alternative energy source in oxidative tissues, including muscle, ketone bodies regulate the metabolism of other substrates, but are cataplerotic. Glycogen can be mobilised for anaplerosis, regenerating Krebs cycle intermediates, thus glycogen availability may affect the hearts' ability to oxidise ketone bodies. We hypothesise that glycogen acts as an anaplerotic substrate for myocardial ketone body oxidation, and thereby affects cardiac exogenous glucose utilisation. We aimed to determine the effects of cardiac glycogen content and ketone body metabolism on glucose utilisation.

Methods Isolated rat hearts were pre-perfused with buffer containing either no substrate (to deplete glycogen) or pyruvate, lactate, glucose and insulin (to augment glycogen content), before switching to $14 \mathrm{C}$-hydroxybutyrate $(\beta \mathrm{HB})$ or $5-3$ hourglucose, plus $11 \mathrm{mM}$ glucose. Timed buffer samples were analysed for $14 \mathrm{CO} 2$ or $3 \mathrm{H}_{2} \mathrm{O}$ to measure $\beta \mathrm{HB}$ oxidation or glycolysis, respectively. Hearts were freeze-clamped for glycogen content.

Results Removal of substrate in the perfusion period significantly decreased myocardial glycogen content $(5.4 \pm 1.6$ vs 43.5 $\pm 5.1 \mu \mathrm{mol}$ glycosyl units/gww). $\beta \mathrm{HB}$ oxidation rate in high glycogen hearts was twice that of low glycogen hearts. Presence of $\beta \mathrm{HB}$ in both high and low glycogen hearts significantly decreased glycolysis from perfusate glucose by $60 \%$ and $38 \%$ respectively (both to $0.29 \mu \mathrm{mol} / \mathrm{gww} / \mathrm{min}$ ).

Conclusion $\beta \mathrm{HB}$ oxidation was increased, and glycolysis from exogenous glucose was decreased, in high glycogen hearts.

\section{P32 MITOCHONDRIA MUST CHOOSE BETWEEN RESISTANCE TO FATTY ACYL COA REGULATION OR RAPID RESPIRATION IN THE TYPE 2 DIABETIC HEART}

Matthew Kerr, Sara Rohling, Maria Sousa Fialho, Colleen Lopez, Damian Tyler, Lisa C Heather. Department of Physiology, Anatomy and Genetics - University of Oxford

\subsection{6/heartjnl-2018-BSCR.37}

Introduction Cardiac metabolism in type 2 diabetes is abnormal, with increased fatty-acid oxidation, and decreased glucose oxidation. We have previously shown a reduction in energetics in the type 2 diabetic heart (20\% decrease in ATP). The control of respiratory rate is mediated primarily $(70 \%)$ by one protein, the adenine nucleotide translocator (ANT). The ANT is inhibited by fatty acyl CoA groups which may relate the reduced energetics in the type 2 diabetic heart to its high fatty acid environment.

Results Palmitoyl-CoA (P-CoA) decreased respiration in control mitochondria by $50 \%$, but in diabetic mitochondria by just $20 \%$. We showed unchanged Vmax, but increased $\mathrm{Km}$ in the presence of $\mathrm{P}-\mathrm{CoA}$, demonstrating competitive inhibition by the fatty acyl CoA group.

Diabetic mitochondria had decreased ADP stimulated, and maximal respiration compared to controls when respired on glutamate, pyruvate and malate. The addition of fatty acids rescued only the ADP stimulated respiratory defect.

Finally, we showed a very strong correlation between decreased respiration, and decreased sensitivity to fatty acyl CoA regulation. Conclusion We have shown that type 2 diabetic mitochondria are resistant to fatty acyl CoA regulation, indicating that this is unlikely to be the cause of energetic dysfunction. We have shown a strong correlation between fatty acid sensitivity and the rate of respiration, two phenomena that are intrinsically linked by the ANT. We propose that changes to ANT kinetics 
in type 2 diabetes provides a strong link between the high fat phenotype, and reduced energetic capacity of type 2 diabetic mitochondria.

\section{P33 PHARMACOLOGICAL STIMULATION OF HIF SIGNALLING AS A POTENTIAL TREATMENT APPROACH FOR THE TYPE 2 DIABETIC HEART}

MdL Sousa Fialho, M Kerr, MS Dodd, C Montes Aparicio, DJ Tyler, LC Heather. Department of Physiology, Anatomy and Genetics, University of Oxford

\subsection{6/heartjnl-2018-BSCR.38}

Introduction In response to ischemia, type 2 diabetic hearts are less able to upregulate anaerobic glycolysis and show poorer contractile function. Pharmacological activation of Hypoxia-Inducible Factor (HIF) may be beneficial for the diabetic heart, as it may promote glycolysis, improve function and tolerance of ischaemia.

Methods Control and type 2 diabetic rats were given five oral doses $(5 \mathrm{mg} / \mathrm{kg}$ daily) of PHD-selective HIF activator BAY853934. Hearts were perfused ex vivo and subjected to low-flow $(0.35 \mathrm{ml} / \mathrm{min} / \mathrm{gww})$ ischaemia-reperfusion (IR). Glycolysis, palmitate oxidation and function were measured throughout. Mitochondrial oxidation was measured in isolated mitochondria using a Clark-type electrode.

Results Diabetic hearts showed decreased rate-pressure product following ischaemia-reperfusion. Pre-treatment with BAY853934 resulted in increased glycolytic rate in diabetic hearts, paired with improved functional recovery post-IR. BAY853934 treated diabetic rats showed increased hematocrit, indicating systemic activation of HIF signalling. Blood glucose levels remained unchanged with treatment despite changes in cardiac glycolytic rate.

Conclusion This study has shown that treatment with HIF activators may provide a novel avenue to improve metabolism and functional recovery in the diabetic heart following ischaemia-reperfusion.

\section{P34 ADENOSINE A1 RECEPTOR ACTIVATION CAN PROTECT THE MYOCARDIUM FROM ISCHAEMIA REPERFUSION INJURY POST REPERFUSION}

J Bhandal, A Hussain, J Buckley, H Maddock. ABES, Coventry University

\subsection{6/heartjnl-2018-BSCR.39}

Introduction/background A1 adenosine receptors (A1AR), have previously been shown to limit injurious infarct development. The purpose of this current study was to determine if delayed activation of A1ARs from 0,15 or $30 \mathrm{~min}$ post-reperfusion can still limit infarct development.

Method Male Sprague-Dawley rats $(300 \pm 50 \mathrm{~g})$ were mounted on the Langendorff system. A1AR agonist, 2-chloro-N cyclopentyl-2'-methyladenosine (2'-MeCCPA) $\quad(0.1 \mathrm{nM}-1 \quad \mathrm{M})$ was administered at the onset of reperfusion as well as 15 and $30 \mathrm{~min}$ post-reperfusion $(10 \mathrm{nM})$ in the presence and absence of the A1AR antagonist 8-cyclopentyl-1,3-dipropylxanthine (DPCPX, $200 \mathrm{nM}$ ) as well as in the presence and absence of Wortmannin $(100 \mathrm{nM})$.

Results Administration of 2'-MeCCPA (1 nM-1 M) at reperfusion significantly reduced infarct size to risk ratio.
Furthermore, delaying the activation of A1AR to 15 or $30 \mathrm{~min}$ post-reperfusion significantly reduced infarct size compared to IR control.

The co-administration of DPCPX $(200 \mathrm{nM})$ with 2'MeCCPA $(10 \mathrm{nM})$ at the onset of reperfusion significantly attenuated the 2'-MeCCPA mediated infarct. The concomitant administration of 2'-MeCCPA with DPCPX at either 15 or $30 \mathrm{~min}$ post-reperfusion also abrogated 2'-MeCCPA induced cardioprotection.

Co-administration of Wortmannin (100 nM) with 2'MeCCPA $(10 \mathrm{nM})$ at the onset of reperfusion also significantly reduced the 2'-MeCCPA mediated infarct size as well as the co-administration at 15 or $30 \mathrm{~min}$ post-reperfusion reversed the cardioprotection.

Conclusion This is the first study to display how 2'-MeCCPA, a highly selective A1AR agonist, when administered at reperfusion, 15 or 30 min post-reperfusion can limit the infarct size development and how the RISK cell signalling pathway is also associated with cardioprotection.

\section{P35 TREATMENT WITH OLEIC ACID AND A PPAR $\alpha$ AGONIST INCREASES FATTY ACID OXIDATION IN BEATING HUMAN INDUCIBLE PLURIPOTENT STEM CELL-DERIVED CARDIOMYOCYTES}

H Al-Siddiqi, CA Lopez, LC Heather, CA Carr. Department of Physiology, Anatomy and Genetics, University of Oxford

\subsection{6/heartjnl-2018-BSCR.40}

Aim Human inducible pluripotent stem cell-derived cardiomyocytes (hiPSC-CM) have an immature phenotype including a glycolytic metabolism. Here we aimed to mature the metabolic status of beating (hiPSC-CM) by targeting the PPAR- $\alpha$ pathway, which regulates the transcription of genes involved in fatty acid metabolism. We cultured hiPSC-CM with the fatty acid Oleic Acid (OA) with or without the PPAR- $\alpha$ agonist WY14643.

Methods hiPSCs cells were differentiated into beating hiPSCCM by treatment with CHIR99021 followed by IWP4. Beating hiPSC-CM were treated with $400 \mathrm{uM}$ OA with or without $120 \mathrm{uM}$ WY14643 for 8 and 24 hours. Oxygen consumption was measured using a Clark-type oxygen electrode, glycolysis was measured by addition of $3 \mathrm{H}$-glucose. Gene expression was measured using qPCR.

Results A significant increase in oxygen consumption and reserve respiratory capacity when respiring on fatty acid was observed in beating iPSC-CM after culture with OA \pm WY14643 for 24 hours compared with untreated and OA-treated hiPSC-CM. OA \pm WY14643 caused a significant increase in expression of carnitine palmitoyltransferase $1 \mathrm{~B}$, medium chain acyl-CoA dehydrogenase and pyruvate dehydrogenase kinase after 8 hours whilst expression of glucose transporters and phosphofructokinase decreased. Addition of OA, but not OA \pm WY14643, increased the rate of O2 respiration on pyruvate and malate but not the reserve respiratory capacity. There was no change in the rate of glycoysis after treatment with OA \pm WY14643 for 24 hours.

Conclusion Treatment with OA and WY14643 for 24 hours increased fatty acid utilisation in beating hiPSC-derived cardiomyoyctes. Funded by the BHF and the Qatar Foundation. 